\title{
Biópsia do miocárdio em cães: acesso minimamente invasivo por cirurgia torácica videoassistida
}

[Myocardial biopsies in dogs: minimally invasive approach by video-assisted thoracoscopy]

\author{
L.G.A. Martins ${ }^{1}$, A.G. Raiser ${ }^{2}$, R.K. Richter ${ }^{3}$, F.V.A. Braga ${ }^{4,5}$, J.C. Rappeti ${ }^{4,5}$, A.N.C. Oliveira ${ }^{6}$ \\ ${ }^{1}$ Pontifícia Universidade Católica do Paraná - Toledo, PR \\ ${ }^{2}$ Centro de Ciências Rurais - UFSM - Santa Maria, RS \\ ${ }^{3}$ Pontifícia Universidade Católica do Paraná - Curitiba, PR \\ ${ }^{4}$ Faculdades Itapiranga - Itapirangas, SC \\ ${ }^{5}$ Universidade Federal de Pelotas - Pelotas, RS \\ ${ }^{6}$ Médica veterinária autônoma
}

\begin{abstract}
RESUMO
Realizou-se uma série de biópsias cardíacas em cães para testar o acesso toracoscópico, utilizando-se oito cães sem raça definida, sob condições de manejo e alimentação adequados. A abordagem à cavidade torácica foi feita por meio de cirurgia torácica videoassistida e o instrumental utilizado para remoção do fragmento de miocárdio foi o convencional em lugar das pinças de videocirurgia. A eficácia do procedimento foi confirmada pelo tempo decorrido entre as incisões, a execução da biópsia e a sutura do tórax, com duração média de 15 minutos. A cirurgia torácica videoassistida para biópsias cardíacas foi considerada segura e adequada, minimizando o desconforto pós-operatório dos pacientes submetidos ao acesso torácico.
\end{abstract}

Palavras-chave: cão, coração, biópsia, videocirurgia

\begin{abstract}
In order to evaluate the thoracoscopy access, biopsies were carried out in eight mongrel dogs maintained under appropriate conditions of handling and feeding. The patients were prepared to aseptic and atraumatic surgery approaching the thoracic cavity by means of video-assisted thoracic surgery (VATS). Myocardial biopsies were carried out with conventional surgical instruments as an alternative to the clamps of video surgery. The effectiveness of the procedure was confirmed by mean time (15 minutes) elapsed from thoracic approach and biopsies obtainment to thoracic wall suture. VATS is a secure and appropriate technique to carry out myocardial biopsies that minimize postoperative discomfort in patients submitted to thoracic approach.
\end{abstract}

Keywords: dog, heart, biopsy, video-assisted surgery

\section{INTRODUÇÃO}

A cirurgia endoscópica é caracterizada como um acesso cirúrgico minimamente invasivo, no qual a visibilização é realizada por meio de um endoscópio. É também denominada de cirurgia laparoscópica, celioscópica, ou pelvioscópica, quando o procedimento envolve o acesso abdominal, e toracoscópica, pleuroscópica, ou cirurgia torácica videoassistida (CTVA), quando o procedimento envolve o acesso torácico (Freeman, 1998).
A toracoscopia foi originalmente introduzida por Jacobeus, em 1910, quando o pesquisador usou um cistoscópio modificado, sem magnificação de imagens, para realizar diagnósticos de tuberculose. $\mathrm{Na}$ última década, o acesso toracoscópico tem registrado uma expansão importante, quer nas possibilidades diagnósticas, quer nas terapêuticas (Jaklitsch, 1996). A partir do início da década de 90, a técnica foi utilizada em maior escala, sendo empregada com êxito na identificação de efusão pleural, de fístulas

Recebido em 19 de fevereiro de 2009

Aceito em 25 de novembro de 2009

E-mail: liege.martins@pucpr.br 
broncopleurais, de lesões esofágicas, de anormalidades pericárdicas, de doenças pulmonares intersticiais difusas e de nódulos pulmonares (Carrillo et al., 1997). Da mesma maneira, o acesso passou a ser utilizado com objetivos terapêuticos, como na ressecção de nódulos pulmonares (Nakajima et al., 2000), excisão e drenagem pericárdica, pleurodesis e biópsias pulmonares (Mack et al., 1992) e drenagem de abscesso mediastinal (Chung e Ritchie, 2000).

Em trabalho de revisão, Remedios e Ferguson (1996) consideraram ainda limitado o acesso toracoscópico na medicina veterinária. Os estudos iniciais restringiram-se à exploração diagnóstica da cavidade (McCarthy e McDermid, 1990). Porém, a toracoscopia vem ganhando terreno também na medicina veterinária, sendo utilizada experimentalmente nas biópsias pulmonares, lobectomias (García et al., 1998), pericardiectomias (Walsh et al., 1999), na redução de hérnias diafragmáticas (Beck, 2004) e suturas de esôfago torácico (Bonfada, 2005) em cães.

A toracoscopia é instrumento importante para determinação de estágios tumorais, visualização de biópsias, avaliação e tratamento de pneumotórax persistente, tratamento de efusão pericárdica e pleural e na avaliação e reparo do trauma intratorácico em pequenos animais (Walton, 2001; Rappeti et al., 2007).

A principal contraindicação da cirurgia torácica videoassistida (CTVA) no trauma diz respeito à instabilidade hemodinâmica do paciente, sendo relacionadas, ainda, suspeita de lesões críticas ao coração e aos grandes vasos, obliteração do espaço pleural, toracotomias anteriores, indicações para toracotomia de emergência e inabilidade para tolerar a ventilação seletiva (Lowdermilk e Naunheim, 2000).

Para acesso e avaliação do músculo cardíaco, é indicada a biópsia cardíaca, que permite determinar o grau de comprometimento do tecido miocárdico por diversas afecções, como a cardiomiopatia dilatada (Eyster, 1993). A obtenção dos fragmentos de miocárdio para análise histopatológica geralmente é feita por biópsia endomiocárdica ou na necrópsia do paciente (Moreira et al., 1998). Conforme a literatura, a avaliação histológica do tecido cardíaco, na necrópsia de animais que morreram de cardiomiopatia dilatada, revela uma série de alterações nas fibras musculares cardíacas distribuídas dispersamente nas paredes dos dois ventrículos (Tidholm et al., 1998). Ademais, a prevalência de fibras com características muito peculiares define não apenas o tipo de cardiomiopatia como também constitui um critério diagnóstico para a doença (Tidholm e Jönsson, 2005).

A CTVA é um procedimento que possibilita visibilização direta da cavidade torácica, permitindo acesso para obtenção de fragmentos de pleura, pulmão, mediastino e pericárdio (Krasna e Mack, 1994; Liu et al., 1994, 2000; Maruyama et al., 2000; Lackner et al., 2000).

Este trabalho visou à realização de um estudo experimental sobre a exequibilidade da biópsia cardíaca por CTVA em cães.

\section{MATERIAL E MÉTODOS}

As biópsias cardíacas foram realizadas em oito cães sem raça definida, sob condições de manejo e alimentação adequados.

O equipamento utilizado na CTVA foi: fonte de luz halógena ${ }^{1}$, cabo de luz de fibra ótica ${ }^{2}$, microcâmera $^{3}$, processador da microcâmera ${ }^{4}$, monitor de vídeo de alta resolução ${ }^{5}$, videocassete $^{6}$, endoscópio rígido de $10 \mathrm{~mm} \varnothing \mathrm{e}$ ângulo de visão de $0^{\circ 7}$ e trocarte de $11 \mathrm{~mm} \varnothing^{8}$. Além desse equipamento, para biópsia, foram utilizados instrumentais de cirurgia convencional: uma pinça de Allis, uma tesoura de Metzembaum e uma pinça de Halsted .

Os pacientes foram preparados para cirurgia asséptica e atraumática com abordagem à cavidade torácica por meio de CTVA. Para acesso, foram feitas três incisões de $1,5 \mathrm{~cm}$, a primeira no oitavo espaço intercostal, no terço

${ }^{1}$ Fonte de luz fria halógena HS 15003, H.Strattner, Rio de Janeiro, RJ.

${ }^{2}$ Cabo de luz 495NCS, H. Strattner, Rio de Janeiro, RJ.

${ }^{3}$ Telecam-Dx 20232101, H. Strattner, Rio de Janeiro, RJ.

${ }^{4}$ Processador Dx 20232101, H. Strattner, Rio de Janeiro, RJ.

${ }^{5}$ Sony, H. Strattner, Rio de Janeiro, RJ.

${ }^{6}$ VCR Philips VR 355 turbo drive, Philips do Brasil, Manaus, AM.

${ }^{7}$ Endoscópio rígido Hoopkins 26033AP, H. Strattner, Rio de Janeiro, RJ

${ }^{8}$ Trocarte $30103 \mathrm{P} / 30103 \mathrm{~K}$, H. Strattner, Rio de Janeiro, RJ. 
médio do tórax, entre a junção costocondral e a costovertebral, onde foi inserido um trocarte de $11 \mathrm{~mm} \varnothing \mathrm{e}$, na sequência, introduzido o endoscópio rígido de $0^{\circ}$ e $10 \mathrm{~mm} \varnothing$. A segunda incisão foi feita no quinto espaço intercostal, numa posição mais dorsal à primeira, e a terceira no quarto espaço intercostal, numa posição mais ventral, próxima à junção costocondral, perfazendo uma configuração triangular na parede costal. Essas duas últimas incisões se prestaram para introdução do instrumental cirúrgico convencional, sob visão direta, com o qual foi realizada a biópsia cardíaca (Fig. 1).

Após a introdução do endoscópio, a cavidade foi inicialmente inspecionada em busca de alterações anatômicas. $\mathrm{O}$ coração foi visibilizado e, após a abertura convencional do pericárdio mediante incisão em forma de "T", foi escolhida a área da parede livre do ventrículo, menos vascularizada, entre os ramos coronarianos descendentes principais, para realização da biópsia (Fig. 2 a, b, c).

O miocárdio foi fixado com pinça cirúrgica de Allis e, em seguida, incisado com tesoura de Metzembaum, sendo obtido um fragmento de aproximadamente $3 \times 3 \mathrm{~mm}$ em cada biópsia. Para controle da hemorragia no miocárdio, foram aplicadas turundas de gaze com auxílio de uma pinça hemostática reta, comprimidos sobre $\mathrm{o}$ local da biópsia por um período de dois a três minutos. Controlada a hemorragia, os coágulos remanescentes foram removidos também com o auxílio das turundas de gaze (Fig. 3 a, b e c).

As incisões toracoscópicas foram suturadas com pontos isolados simples e fio mononáilon 3-0. A pressão negativa intratorácica foi restabelecida utilizando-se um cateter acoplado a uma seringa de $60 \mathrm{~mL}$ e uma torneira de três vias, por meio de aspiração do ar do interior da cavidade. No pósoperatório, os pacientes dos dois grupos receberam curativos na ferida cirúrgica com iodo povidine, e como analgésicos flunixin meglumine $\left(1,1 \mathrm{mg} \mathrm{kg}^{-1}\right)^{9}$ intramuscular SID, por três dias, e morfina $\left(0,5 \mathrm{mg} \mathrm{kg}^{-1}\right)^{10}$ QID, por 24 horas.

\footnotetext{
${ }^{9}$ Banamine - Ind. Quim. Farm. Schering Plough SA. Divisão Veterinária.

${ }^{10}$ Dimorf 10mg mL $\mathrm{mL}^{-1}$ - Cristália Produtos Químicos

Farmacêuticos Ltda. Itapira SP.
}

Depois da biópsia, os animais permaneceram em observação por uma semana e, após a retirada dos pontos da pele, foram encaminhados para adoção. A pesquisa foi aprovada pelo Comitê de Ética da Universidade Federal de Santa Maria.

\section{RESULTADOS E DISCUSSÃO}

A CTVA na medicina veterinária tem avançado como um método para diagnóstico e tratamento de diversas desordens intratorácicas (Richter et al., 2007) e também permite acesso para retirada de fragmentos e biópsias conforme citaram Krasna e Mack (1994), Liu et al. (1994, 2000), Lackner et al. (2000) e Maruyama et al. (2000). Assim, realizou-se este experimento com vistas à aplicação clínica da técnica no controle pré e pós-operatório de intervenções cirúrgicas cardíacas, bem como no auxílio diagnóstico de afecções cardíacas degenerativas progressivas como a cardiomiopatia dilatada.

Observou-se que não apenas a obtenção de fragmentos de pleura, lobos pulmonares e pericárdio, como relatado na literatura (Walton, 2001; Willard, 2002; De Zoppa et al., 2008), são viáveis por meio da CTVA, mas também do próprio miocárdio, sem riscos de hemorragia profusa, pois, no momento da retirada do fragmento de miocárdio, a simples compressão local com gaze por dois a três minutos foi suficiente para conter o sangramento local.

O uso de instrumental cirúrgico convencional na realização das biópsias foi considerado satisfatório, tornando o procedimento mais confortável para o cirurgião que trabalhou a poucos centímetros da incisão, sem a necessidade de trocarteres. Salvo pela necessidade de serem pinças e tesouras um pouco mais longas, não se encontrou dificuldade na apreensão das estruturas no interior da cavidade torácica e, além disso, foi possível sentir bastante segurança durante as manobras, pela proximidade das mãos do cirurgião com as estruturas manipuladas dentro do tórax. $\mathrm{O}$ procedimento tornou-se menos oneroso, uma vez que o instrumental de videocirurgia é caro e descartável, conforme também consideraram Liu et al., (1994), Yim (1995), Isakow et al. (2000) e Liu et al. (2000) e em seus trabalhos de videotoracoscopia utilizando instrumental cirúrgico convencional. 


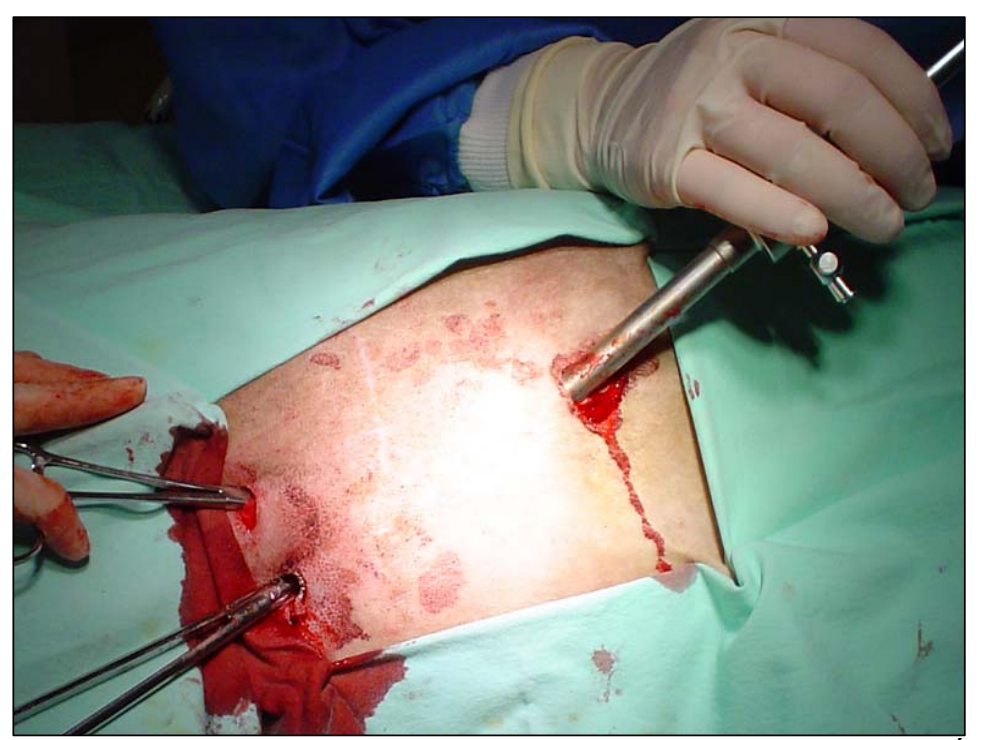

Figura 1. Cão. Distribuição dos portais de acesso ao tórax para biópsia cardíaca. Ótica ao lado direito e portais com os instrumentais cirúrgicos convencionais já posicionados ao lado esquerdo.

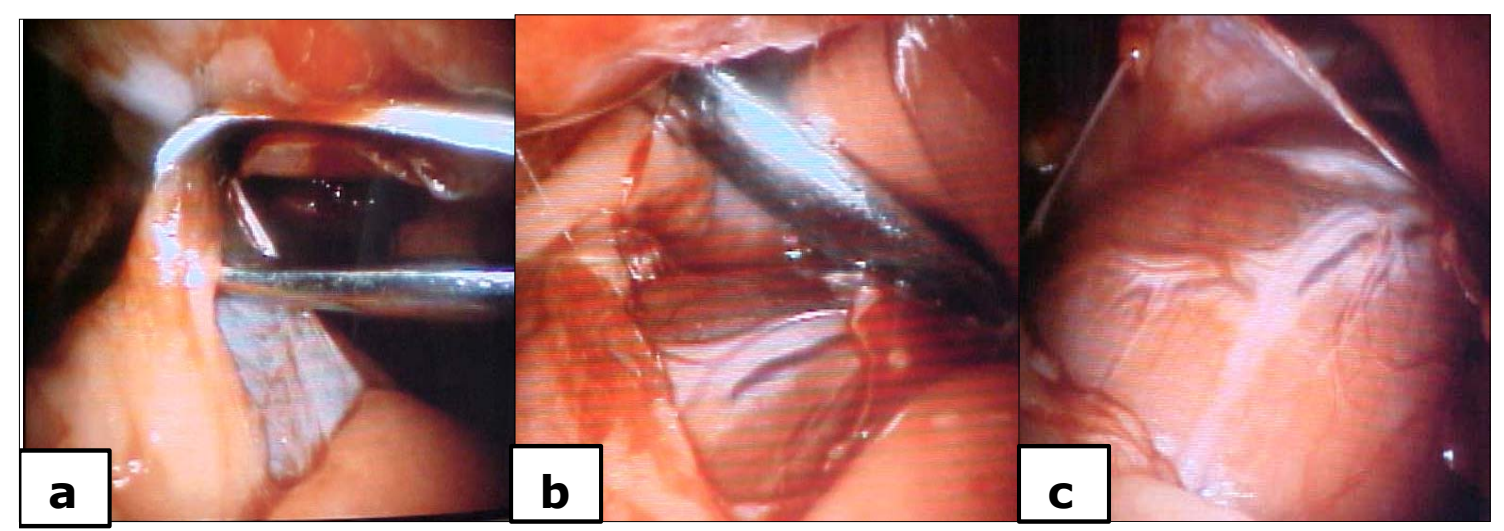

Figura 2. Cão. Acesso cardíaco para biópsia: fixação do pericárdio (a), incisão do pericárdio (b), exposição da área ventricular livre menos vascularizada, entre os ramos coronarianos descendentes principais, para realização da biópsia (c).

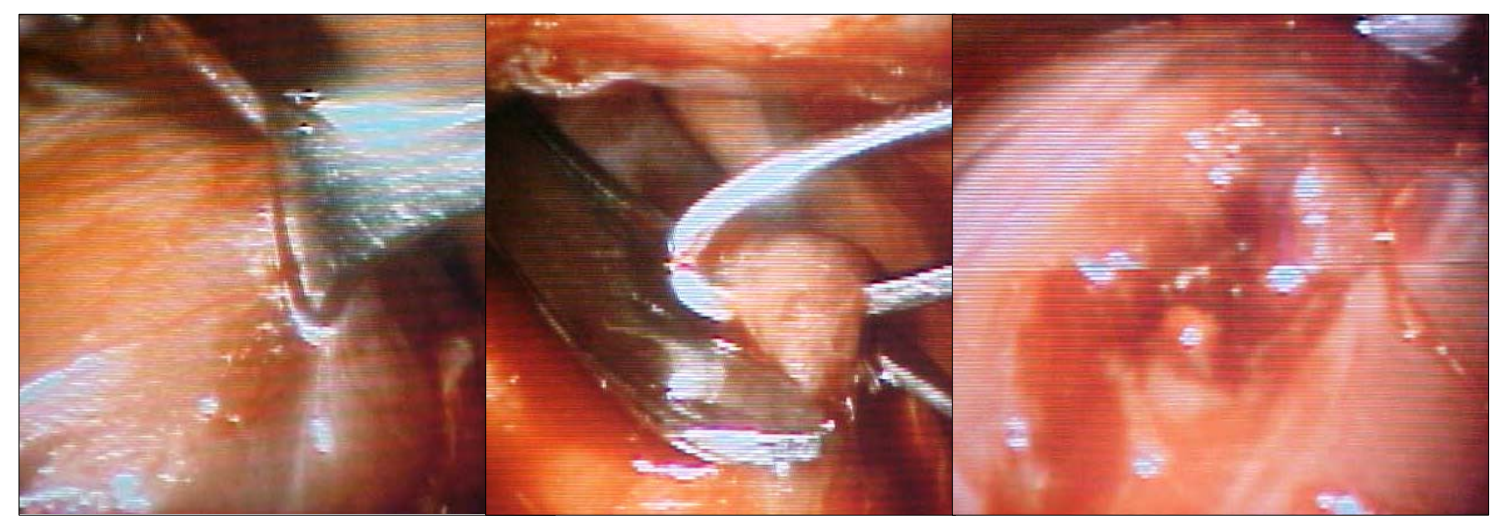

Figura 3. Cão. Fixação do miocárdio com pinça de Allis (a), incisão do miocárdio com tesoura de Metzembaum (b), aspecto do leito miocárdico após a biópsia e hemostasia (c). 
Constatou-se que a cirurgia minimamente invasiva foi adequada, levando-se em consideração o conhecido desconforto pósoperatório dos pacientes submetidos à toracotomia. Neste experimento, comprovaramse as vantagens dessa abordagem, pois os animais, já no primeiro dia de pós-operatório, saíam do canil para passear sem sinais de dor ou dispneia e alimentavam-se normalmente. Isto está de acordo com os achados de Kaseda et al. (2000), que consideraram o prognóstico da função pulmonar depois da videotoracoscopia ou da CTVA muito mais favorável em comparação com a toracotomia. Liu et al. (2000) e Chan et al. (2001) também comentaram que as taxas de incidência de neuralgia pós-operatória são baixas em relação à toracotomia convencional, pois o trauma de acesso é minimizado pela abordagem, já que os pontos de incisão são muito pequenos, o tempo de internação dos pacientes é menor, portanto mais econômico, e também é mais cosmético, pois as cicatrizes das pequenas incisões tornam-se quase imperceptíveis .

A eficácia do procedimento também foi confirmada pelo tempo decorrido entre: as incisões no tórax, a execução da biópsia e a sutura do tórax, com duração média de 15 minutos, muito inferior ao tempo de abordagem e fechamento do tórax em uma toracotomia convencional.

\section{CONCLUSÃO}

A cirurgia torácica videoassistida é uma abordagem segura e adequada para biópsia do miocárdio em cães; a abordagem minimamente invasiva para esse procedimento é recomendada por ser rápida e permitir rápida recuperação dos pacientes; o material cirúrgico convencional é apropriado para este tipo de procedimento.

\section{REFEÊNCIAS BIBLIOGRÁFICAS}

BECK, C.A.C.; PIPPI, N.L.; BRUN, M.V. et al. Toracoscopia nas hérnias diafragmáticas: estudo experimental em cães. Cienc. Rural, v.34, p.1857-1863, 2004.

BONFADA, A. T. Cirurgia torácica videoassistida sem intubação seletiva com acesso modificado para sutura do esôfago caudal em cães. 2005. 46f. Dissertação (Mestrado) - Universidade Federal de Santa Maria, Santa Maria.
CARRILLO, E.H.; HENIFORD, B.T.; ETOCH, S.W. et al. Video-assisted thoracic surgery in trauma patients. J. Am. Coll. Surg., v.184, p.316$323,1997$.

CHAN, P.; CLARKE, P.; FRACS, F.J.D. et al. Efficacy study of video-assisted thoracoscopic surgery pleurodesis for spontaneous pneumothorax. Ann. Thorac. Surg., v.71, p.452454, 2001.

CHUNG, D.A.; RITCHIE, A.J. Videothoracoscopic drainage of mediastinal abscess: an alternative to thoracoscopy. Ann. Thorac. Surg., v.69, p.1573-1574, 2000.

DE ZOPPA, A.L.V.; ALVARENGA J.; CRUZ R.S.F. et al. Toracoscopia aplicada à ressecção de fragmento pulmonar com o auxílio de sutura mecânica em equinos. Arq. Bras. Med. Vet. Zootec., v.60, p.559-565, 2008.

EYSTER, G.E. Cardiac disorders. In: SLATTER, D. (Ed.). Textbook of small animal surgery. 2.ed. Philadelphia: WB. Saunders, 1993. v.1, p.856-857.

FREEMAN, L.J. Introduction. In: Veterinary endosurgery. Saint Louis: Mosby, 1998.

GARCIA, F.; PRANDI, D.; PENA, T. et al. Examination of the thoracic cavity and lung lobectomy by means of thoracoscopy in dogs. Can. Vet. J., v.39, p.285-291, 1998.

ISAKOW, K.; FOWLER, D.; WALSH, P. Video-assisted thoracoscopic division of the

ligamentum arteriosum in two dogs with persistent aortic arch. J. Am. Vet. Med. Assoc., v.217, p.1333-1336, 2000.

JAKLITSCH, M.T. Video-assited techniques in thoracic surgery. In: LOUGHLIN, K.R.; BROOKS, D.C. Principles of endosurgery. Cambridge: Blackwell Science, 1996. p.230-250.

KASEDA, S.; AOKl, T.; HANGAl, N. et al. Better pulmonary function and prognosis

187 with videoassisted thoracic surgery than with thoracotomy. Ann. Thorac. Surg., v.70, p.16441646, 2000.

KRASNA, M.J.; MACK, M.J. Atlas of thoracoscopic surgery. Saint Louis: Quality Medica1, 1994. p.216. 
LACKNER, R.P.; HUGHES, R.; ANDERSON, L.A. et al. Video-assisted evacuation of empiema is the preferred procedure for managemente of pleuralspace infections. Am. J. Surg.,v.179, p.27-30, 2000.

LIU, H.P.; CHANG, C.H.; LIN, P.J. et al. Thoracoscopic removal of intrathoracic neurogenic tumors: A combined chinese experience. Ann. Surg., v.232, p.187-190. 2000.

LIU, H.P.; CHANG, C.H.; LIN, P.J. et al. Videoassisted thoracic surgery. J. Thorac. Surg., v.108, p.834-840, 1994.

LOWDERMILK, G.A.; NAUNHEIM, K.S. Thoracoscopic evaluation and treatment of thoracic trauma. Surg. Clin. North Am., v.80, p.1535-1542, 2000.

MACK, M.J.; ARONOFF, R.J.; ACUFF, T.E. et al. Present role of thoracoscopy in the diagnosis and treatment of diseases of the chest. Ann. Thorac. Surg., v.54, p.403-409, 1992.

McCARTHY, T.C.; McDERMID, S.L. Thoracoscopy. Vet. Clin. N. Am. Small Anim. Pract., v.20, p.1341-1352, 1990.

MARUYAMA, R.; OKA, T.; ANAI, H. Videoasisted thoracoscopic treatment for spontaneus pneumothorax as two-day surgery. Am. J. Surg., v.180, p.171-173, 2000.

MOREIRA, L.F.P.; STOLF, N.A.G.; BOCCHI, E. et al. Existe lugar para a ventriculectomia parcial esquerda no tratamento da cardiomiopatia dilatada? Rev. Bras. Cir. Cardiovasc., v.13, p.8999, 1998.

NAKAJIMA, J.; TAKAMOTO, S.; TANAKA $M$. et al. Thoracoscopic resection of the pulmonary aspergilloma. Chest, v.118, p.14901495, 2000.
RAPPETI, J.C.S.; PIPPI N. L.; BRAGA F.V.A. et al. Pós-operatório de homoimplante de costela avaliado por toracoscopia paraxifoide transdiafragmática modificada em gatos. Cienc. Rural, v.37, p.1355-1359, 2007.

REMEDIOS, A.M.; FERGUSON, J. Minimally invasive surgery: laparoscopy and thoracoscopy in small animals. Compend. Contin. Educ. Pract. Vet., v.18, p.1191-1199, 1996.

RICHTER, R.K.; PIPPI, N.L.; ROCHA, G.S. et al. Cirurgia torácica videoassistida (CTVA) na correção de ducto arterioso patente. Estudo experimental em cães. Arch. Vet. Sci., v.12, p.3540, 2007.

TIDHOLM, A.; HÄGGSTRÖM, J.; JÖNSSON, L. Prevalence of attenuated wavy fibers in myocardium of dogs with dilated cardiomyopathy, J. Am.Vet. Med. Assoc., v.212, p.1732-1734, 1998.

TIDHOLM, A.; JÖNSSON, L. Histologic characterization of canine dilated cardiomiopathy. Vet. Pathol., v.42, p.1-8, 2005.

WALSH, P.J.; REMEDIOS, A.M.; FERGUSON J.F. et al. Thoracoscopic versus open partial pericardectomy in dogs: comparison of postoperative pain and morbidity. Vet. Surg., v.28, p.472-479, 1999.

WALTON, R.S. Video-assisted thoracoscopy. Vet. Clin. N. Am. Small Anim. Pract., v.31, p.729-759, 2001.

WILLARD, M.D. Endoscopy ofbody cavities. In: FOSSUM, T.W. Small animal surgery. 2.ed. Saint Louis: Mosby, 2002. p.130-132.

YIM, A.P.C. Minimizing chest wall trauma in video-assisted thoracic surgery. J. Thorac. Cardiov. Surg., v.109, p.1255-1256, 1995. 